\title{
TOPOLOGY DESIGN OF STRUCTURES, MATERIALS AND MECHANISMS - STATUS AND PERSPECTIVES
}

\author{
Martin P. Bendsøe \\ Department of Mathematics \\ Technical University of Denmark \\ DK-2800 Lyngby, Denmark \\ M.P.Bendsoe@mat.dtu.dk
}

\section{INTRODUCTION}

The field of variable-topology shape design in structural optimization has its origins in theoretical studies of existence of solutions in variational problems, in particular shape optimization problems, and in studies in theoretical material science of variational bounds on material properties. Progress in computational methods and the ever increasing computer power has oriented the area towards applications, with significant developments being achieved over the last decade, leading to a fairly widespread use of the methodology in industry.

In this short paper we outline some of the basic ideas and methods of existing methods, but it is not our purpose to cover all work and approaches in this field. Instead we refer to existing literature containing rather comprehensive surveys, see e.g., $[7,8,31]$. Moreover, note that reference is mostly made to recent papers that include bibliographies useful for on overview of the area. Thus the presentation does not try to present a complete historical perspective.

The area of computational variable-topology shape design of continuum structures is presently dominated by methods which employ a material distribution approach for a fixed reference domain, in the spirit of the so-called 'homogenization method' for topology design $([3,9])$. That is, the geometric representation of a structure is similar to a grey-scale rendering of an image, in discrete form corresponding to a raster representation of the geometry on a fixed reference domain. The physics of the problem is also represented by boundary conditions and forcing

The original version of this chapter was revised: The copyright line was incorrect. This has been corrected. The Erratum to this chapter is available at DOI: 10.1007/978-0-387-35514-6_15 
terms defined on this fixed reference domain, much in analogy to fictitious domain methods for FEM analysis.

One can normally distinguish between three versions of raster based geometry models for continuum topology optimization. The basic problem is an unrestricted '0-1' integer design problem (generalized shape optimization), that is, a design specifies unambiguously whether there is solid material or void at every point in a candidate design region. Otherwise, there are no restrictions on the shape. Unfortunately, in general, this class of problems is ill-posed in the continuum setting (cf., $[11,20])$. Well-posed problems can be obtained by either extending the space of admissible solutions to obtain their relaxed versions, usually by incorporating microstructure (see, e.g., [3]), or by restricting the space of admissible solutions. The latter can be accomplished by enforcing an upper bound on the perimeter of the structure (see [28], and references therein), by imposing constraints on the slopes of the parameters defining geometry (see [29], and references therein), by the introduction of a filtering function limiting the minimum scale (see $[10,36]$ for an overview), or one can introduce a ground structure with a fixed number of design degrees of freedom (like a fixed mesh for design). Here the first three restriction methods are well-posed in the setting of a continuum description of the design problem, while in the latter case existence relies entirely on the finite dimension.

Relaxation usually yields a set of continuously variable design fields to be optimized over a fixed domain, so the algorithmic problems associated with the discrete 0-1 format of the basic problem statement are circumvented. The continuum relaxation approach can be very involved theoretically (see for example $[1,13]$ ) and much work is still needed in this area.

The restriction approach leads to 'classical designs' and there is no ambiguity as to the physical modelling (local material response is determined solely by the presence or absence of the given solid material). However, the major challenge is the solution of a large-scale integer programming problem. Due to the high cost of function calls for these problems, solving the 0-1 formulation directly, for example by genetic algorithms or simulated annealing, is, in general, not viable for very large-scale problems, and this is an area that should have more focus in the coming years (see also below). Another - and the most commonly used - approach is to replace the integer variables with continuous variables and then introduce some form of penalty that steers the solution to discrete $0-1$ values. A key part of these methods is the introduction of interpolation functions (often interpreted as material densities) that express various physical quantities (e.g., material stiffness, cost, etc.) as 
a function of the continuous variables. Moreover, geometric properties also require suitable interpretation. Although there is a strong resemblance to the relaxed formulations, it is important to recognize that the continuous format is then merely part of a computational strategy which does not alter the ultimate goal of solving an integer problem, i.e., to obtain a black-and-white design.

\section{BASIC PROBLEM STATEMENT}

In continuum topology design we seek the optimal distribution of material in a fixed reference domain $\Omega$ in $\mathbf{R}^{\mathbf{2}}$ or $\mathbf{R}^{\mathbf{3}}$, with the term 'optimal' being defined through choice of objective and constraint functions. The objective and constraint functions involve some kind of physical modelling that provides a measure of efficiency within the framework of a given area of applications, here structural mechanics. Here we thus consider a mechanical element as a body occupying a domain $\Omega^{m}$ which is part of $\Omega$ on which applied loads and boundary conditions are defined (this reference domain is often called the ground-structure). Referring to the reference domain $\Omega$ we can define an example problem as a minimization of force times displacement, over admissible designs and displacement fields satisfying equilibrium (the minimum compliance problem):

$$
\min _{C, u} \int_{\Omega} f u d \Omega
$$

subject to:

$$
\begin{aligned}
\int_{\Omega} C_{i j k l}(x) \epsilon_{i j}(u)(x) \epsilon_{k l}(v)(x) d \Omega & =\int_{\Omega} f v d \Omega \\
C_{i j k l} & \in E_{\mathrm{ad}}
\end{aligned}
$$

Here the equilibrium equation is written in its weak, variational form, with $U$ denoting the space of kinematically admissible displacement fields, $u$ the equilibrium displacement, $f$ the forces, and $\epsilon(u)$ linearized strains. The rigidity tensor $C_{i j k l}$ is the design variable of our problem and various definitions of the set of admissible rigidity tensors is what distinguishes various settings for the design problem. This type of problem is what is often labelled as a problem of 'control in the coefficients' (c.f., [22]), here with the controls entering in the high order part of the governing differential operator.

A classical variant of problem (1) is the so-called variable thickness problem, where the set of admissible designs is defined through a thick- 

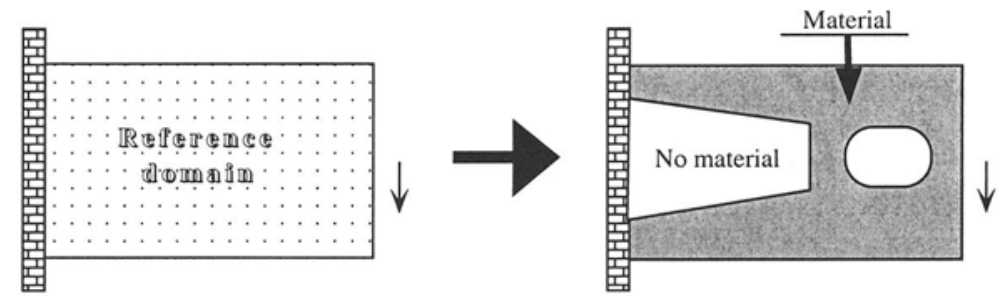

Figure 1 The generalized shape design problem of finding the optimal material distribution.

ness function $h$ :

$$
\begin{aligned}
& C_{i j k l}(x)=h(x) C_{i j k l}^{0}, \quad h \in L^{\infty}(\Omega), \\
& \int_{\Omega} h d \Omega=V, \quad 0 \leq h_{\min } \leq h \leq h_{\max }
\end{aligned}
$$

for given material properties $C_{i j k l}^{0}$ and given volume $V$. This problem is rather unique, as one here has existence of solution in the 'naive' setting, i.e., in the formulation which immediately springs to mind when modelling the problem (see [27] and references therein).

For a topology design setting, defined through designs given as domains $\Omega^{m}$ of material points, the admissible designs are defined by a point-wise volume fraction of a given material, and this density can only attain the values zero or one (a black-and-white design), c.f., Figure 1:

$$
\begin{aligned}
& C_{i j k l}(x)=\Theta(x) C_{i j k l}^{0}, \\
& \Theta(x)=1, x \in \Omega^{m} ; \quad \Theta(x)=0, x \in \Omega \backslash \Omega^{m} \\
& \operatorname{Vol}\left(\Omega^{m}\right)=\int_{\Omega} \Theta(x) d \Omega=V
\end{aligned}
$$

Here existence of solutions usually require further consideration as to the modelling of the problem. Loosely speaking, materials with a structural hierarchy allow for stiffer structures, as seen in nature in bone, wood, etc. and used in composite structures (see Figure 2). This can lead to a lack of existence of solutions, as such composites can be constructed as limiting sequences of designs defined by (3); however, composites are not covered by (3) (if $C_{i j k l}^{0}$ is isotropic, the material in (3) is at any point isotropic, but a composite will usually be anisotropic). One says that the set designs is not closed under G-convergence (or H-convergence) (cf., e.g., $[20,23])$.

One technique to obtain a well-posed problem, as mentioned in the introduction, is to introduce a constraint $G\left(\Omega^{m}\right) \leq \gamma$ on for example 


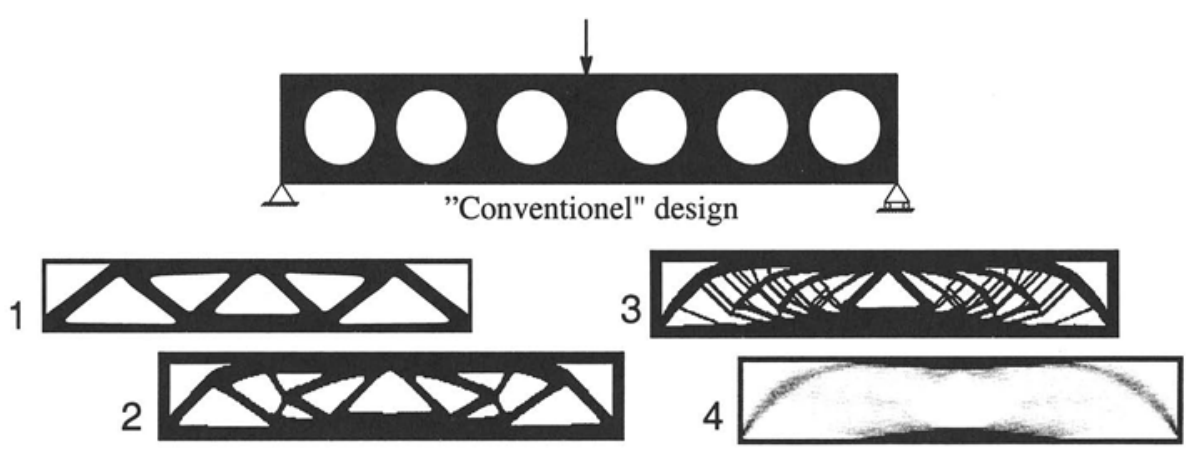

Figure 2 A 'traditional' design of a beam with holes, as seen in aircraft etc., compared with elements of a minimizing sequence of designs with finer and finer scales, with the limit (4) consisting of composites in large areas. Note that even design (1), which is an optimal topology within a limit on geometric complexity, is $40 \%$ stiffer than the design with round holes, for the same amount of material.

the perimeter of the domain, thus limiting the geometric complexity of the domain $\Omega^{m}$ so that a structural hierarchy cannot be generated (existence still requires a formal proof, as seen in $[4,10]$ ). If the geometric constraint is not imposed in (1), the continuum problem is ill-posed, and an alternative for obtaining existence of solutions is to extend the problem to its relaxed form using composites formed by mixing void and the given material. This changes the nature of the problem from one of seeking black-and-white designs to a situation where 'grey-scale' structures may also appear.

Problem (1) with design set (3) (regularized through a geometric constraint) is a large scale discrete optimization problem and it has in this form only recently been handled with success computationally ([6]). The most popular method for solving (1), and an approach that has been extremely successful for many applications, is to consider formulations in terms of continuous variables, with the goal of using derivative based mathematical programming algorithms. This means that one changes the model for the material properties to a situation where the volume fraction is allowed to take on any value between zero and one. It may also involve finding an appropriate method for limiting geometric complexity, for example, exchanging the total variation of a density for the perimeter of a domain. This means that one pursues the optimal black-and-white design through an iterative computational procedure which at intermediate steps operate with 'grey' designs. This can be rather confusing, as the computational procedures for the geometrically constrained black- 
and-white design problem and for the relaxed formulation, cunningly so, can be very similar.

\section{A FRAMEWORK FOR COMPUTATIONS}

Computational means are central for solving problems of the type (1). Typically a finite dimensional version of the problem is generated first, normally with finite element approximations of displacement fields and design variables. This leads to a non-linear, non-convex, large scale optimization problem of the generic form

$$
\text { inf } \begin{aligned}
& p^{T} u \\
& K(D) u=p \\
& \sum D_{i}=V, \quad 0<D_{\min } \leq D_{i} \leq D_{\max },
\end{aligned}
$$

where $u$ here is the vector of nodal displacements and $D$ the design variables, entering the equilibrium equation through the stiffness matrix $K$. This problem could be solved directly via large-scale general purpose mathematical programming techniques or by use of specially developed algorthms that utilizes the problem structure, as seen in 4 . Such techniques treat both displacements and design variables as independent variables and the equilibrium equation is solved by the optimization code (an example of this can be found in [41]).

Such an approach is not very common, as one traditionally rewrites the problem as a problem in the design variables only, as

$$
\begin{array}{ll}
\inf & F(D) \\
& \sum D_{i}=V \\
& 0<D_{\text {min }} \leq D_{i} \leq D_{\text {max }},
\end{array}
$$

where the equilibrium equation is considered as a function-call:

$$
\begin{aligned}
& F(D)=p^{T} u, \\
& \text { where } u \text { solves } K(D) u=p .
\end{aligned}
$$

If function gradients are required by the optimization algorithm, sensitivity analysis becomes a central theme, i.e., one should devise efficient means to compute the derivatives of functions involving the displacements. This usually involves the so-called adjoint system, as also known from control theory. Here, provided the stiffness matrix is positive definite, we have here the simple expression (the computation of the derivative of the stiffness matrix with respect to design is simple for topology 
design):

$$
\begin{aligned}
& \frac{\partial F}{\partial D_{i}}=-u^{T} \frac{\partial K}{\partial D_{i}} u, \\
& \text { where } u=(K(D))^{-1} p .
\end{aligned}
$$

However, in many cases this analysis is not so simple. Sensitivity analysis is actually, in its own right, an important area of computer aided design, as it provides information on changes in performance with respect to design variables, see for example papers in [18].

In many situations it is convenient to operate on (5), as one here treats the equilibrium analysis as a function call, as represented by, e.g., commercial software packages for finite element analysis. Moreover, in many design problems, such as shape design via a CAD definition of boundaries, the number of design variables is much smaller than the number of degrees of freedom of the displacement, and an optimization code used for (5) will thus treat a moderately sized problem (with 'expensive' function calls). However, for topology design, even problem (5) is largescale; but in many cases one can formulate relevant problems involving only a moderate number of constraints, which are not box-constraints (as in the model problem used here). Thus dual methods have become popular, and especially techniques based on separable, convex approximations (CONLIN, see [16], and MMA, see [39]) have been found to be particularly effective.

We close this brief section on computational issues by noting that the problem at hand is a two field problem which may develop checkerboards in computations (as for a Stokes flow problem). Constraints limiting geometric complexity usually removes such effects (for a fine enough mesh), but in a relaxed setting (with existence of solutions, but no geometric constraints) special care must be taken to avoid such 'polluted' results. See [36] for an overview and further discussion.

\section{HOMOGENIZATION MODELS WITH ANISOTROPY}

The initial work on numerical methods for topology design of continuum structures was based on using composite materials as the basis for describing varying material properties in space ([9]). This approach was inspired by theoretical studies on generalized shape design in conduction and torsion problems and by numerical and theoretical work related to plate design (see, e.g., $[11,17,23]$ ). Initially, composites consisting of square or rectangular holes in periodically repeated square cells were used for planar problems. Later so-called ranked laminates (layers) have become popular, both because analytical expressions of their effective 
properties can be given and because investigations proved the optimality of such composites, in the sense of bounds on effective properties (for an overview, see for example [2]). Also, with layered materials existence of solutions to the minimum compliance problem for both single and multiple load cases is obtained, without any need for additional constraints on the design space (e.g., without constraints on the geometric complexity), and thus interpolation and relaxation are both provided for. However, the relaxed models seldom result in black-and-white designs in themselves. For all the models mentioned here, homogenization techniques for computing effective moduli of materials play a central role. Hence the use of the phrase 'the homogenization method for topology design' for procedures involving this type of modelling.

The homogenization method for topology design involves working with orthotropic or anisotropic materials. This adds to the requirements of the finite element analysis code, but the main additional complications is the extra design variables required to describe the structure. Thus, a so-called rank-2 microstructure with two orthogonal layers of material (at two scales and in dimension two) require three distributed variables, as the material properties at each point of the structure will depend on two size-variables characterizing the layer thicknesses and on one variable characterizing the angle of rotation of the material axes (the axes of the layers).

\section{THE SIMP MODEL}

Complementing the use of the homogenization method, where anisotropic composites are a priori accepted as part of the design space, a popular method to model material properties which are isotropic at intermediate densities is the so-called penalized, proportional fictitious material SIMP-model (SIMP: Solid Isotropic Material with Penalization), see for example [8] for an overview. In this model a continuous variable $\rho$ is introduced, with $0 \leq \rho \leq 1$, resembling a density of material by the fact that the volume of the structure is evaluated as

$$
V=\int_{\Omega} \rho(x) d \Omega
$$

The relation between this density and the material tensor $C_{i j k l}$ in the equilibrium analysis is written as

$$
C_{i j k l}(\rho)=\rho^{p} C_{i j k l}^{0}, \quad p>1,
$$

where the given material has stiffness given by $C_{i j k l}^{0}$. The interpolation 9 satisfies that $C_{i j k l}(0)=0$ and $C_{i j k l}(1)=C_{i j k l}^{0}$, meaning that if a 


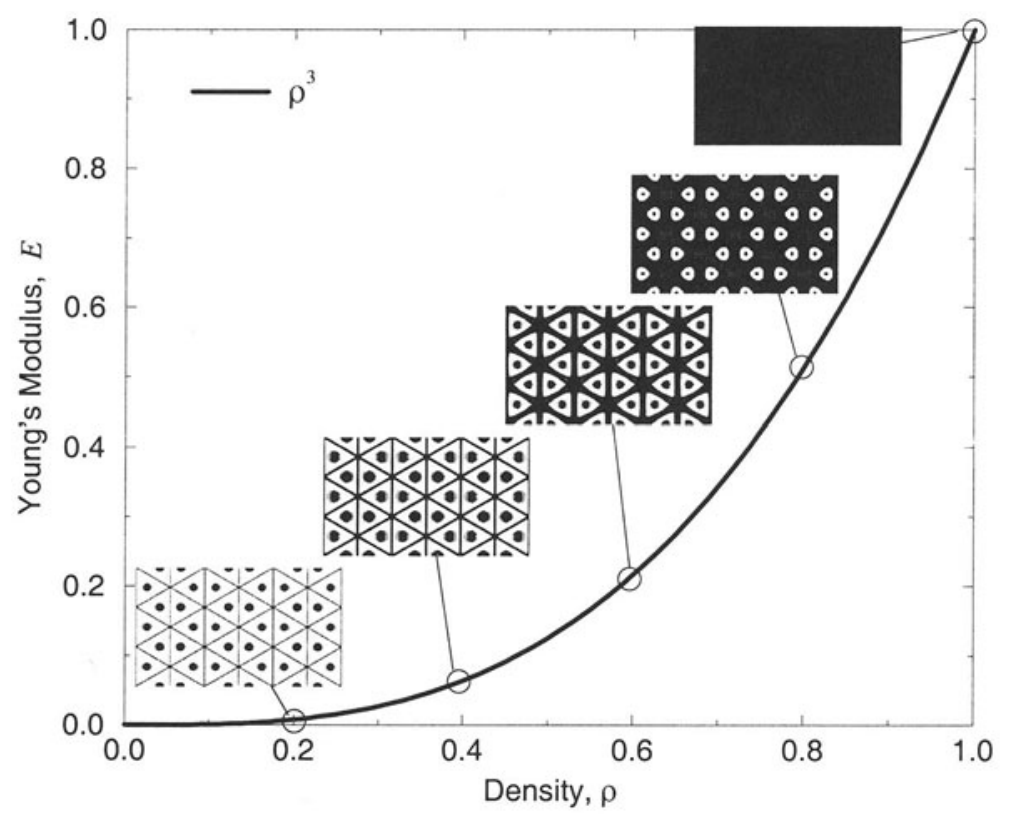

Figure 3 Microstructures of material and void realizing the material properties of the SIMP model with $p=3$ (Poisson's ratio is 0.33 ). As stiffer material microstructures can be constructed from the given densities, non-structural areas are seen at the cell centers. See [8].

final design has density zero and one in all points, this design is a blackand-white design for which the performance has been evaluated with a correct physical model. For problems where the volume constraint is active, experience shows that optimization does actually result in such designs if one chooses the power $p$ sufficiently big (in order to obtain true ' $0-1$ ' designs, $p \geq 3$ is usually required). The reason is that for such a choice one imposes a penalization on intermediate densities (volume is proportional to $\rho$ but stiffness is less than proportional).

For the SIMP interpolation 9 it is not immediately apparent that areas of grey can be interpreted in physical terms. However, it turns out that under fairly simple conditions on $p$, any stiffness used in the SIMP model can be realized as the stiffness of a composite made of void and an amount of the base material corresponding to the relevant density, see Figure 3. Thus using the term 'density' for the interpolation function $\rho$ is quite natural. The geometries in Figure 3 represent periodic composites with repetitive cells, obtained through the methodology of inverse homogenization (material design) described in [33]. This method employs topology design (using SIMP!) for the cell of the microstructure 


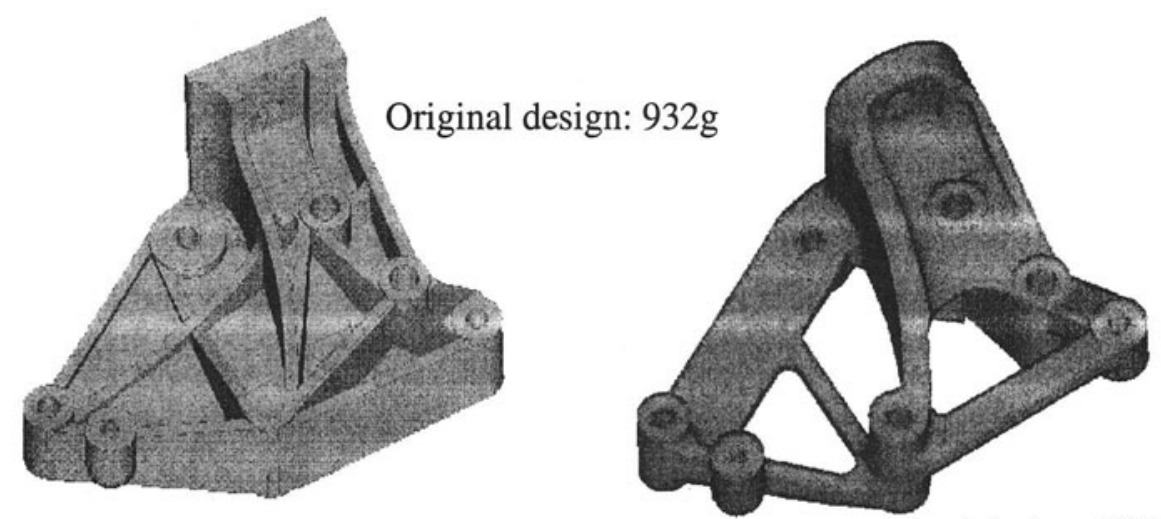

Final design: $732 \mathrm{~g}$

Figure 4 Design of a bracket for an automobile application. Original design and design achieved by using topology design in the initial design phase. By courtesy of Altair Engineering.

to obtain specified material properties of the composite, making the dog bite its tail.

\section{APPLICATIONS}

Computational means for topology design have now been developed into reliable tools for considering such problems involving stiffness (including multiple load problems) and vibration criteria (as a reinforcement problem, see, e.g., [12]). The efficiency of topology design as a tool in the initial phase of a design process and the appearance of commercial software has lead to a broad acceptance of the method in industry, , see, e.g., [5, 32, 40] (see also Section 8). Research has also lead to considering extended classes of problems, encompassing more involved settings for the physical model, for the optimization formulation, as well as for the design parametrization. Thus non-linear behaviour (large displacements, non-linear material behaviour), local constraints (i.e., constraints imposed on all points in the domain) as well as multi-material problems are covered in current research. The areas of applications are also being extended, research moving into such fields as design of materials, see Figure 5, and design of multiple physics devices (like MEMS, MicroElectro-Mechanical-Systems), see Figure 6. For example literature we refer to $[14,19,24,25,34,37]$.

It is important in this context to note that direct links between specific classes of composites and proofs of existence for many of these extended 

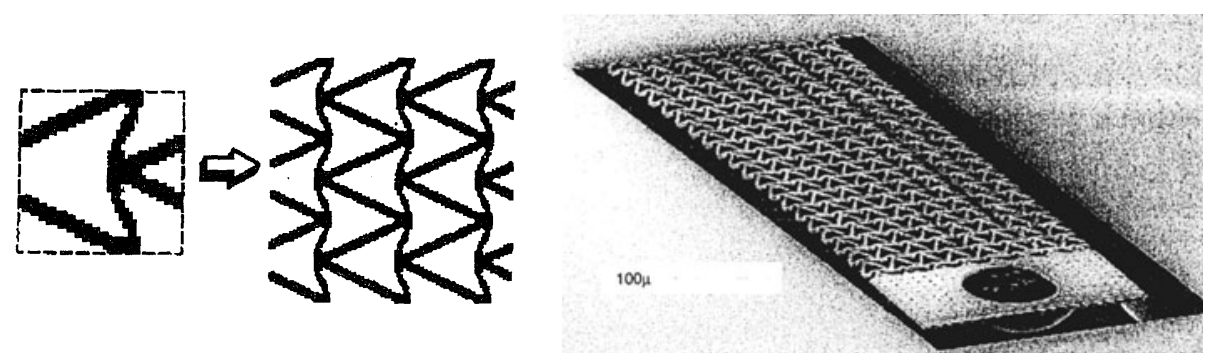

Figure 5 Design of a periodic composite with negative Poisson ratio. Topology optimization is applied to the unit cell of periodicity, and the effective parameters of the composite are computed via homogenization. The right-hand picture shows a micro-device representing the composite. By courtesy of Ole Sigmund, Ulrik Darling Larsen and Siebe Boustra, see also [21, 33].

problems have yet to be discovered, i.e., it is not known for these problems what composites will make relaxation intrinsic in such an approach. The emphasis in the field is currently on modelling and on the development of computational means, mostly relying on restriction methods as an underlying implicit guarantee of well- posedness. Thus, in the context of theory, the pace of implementations for new problem types has far overtaken the full mathematical understanding of the interplay between methods for obtaining 'classical' solutions and methods for deriving relaxed problem settings, and here lies an immense challenge for the future. For a mathematician there is thus an abundance of (hard) problems to study.

For exemplification of modelling consideration we will here briefly outline some aspects of treating multiple physiscs problems. The phrase 'multiple physics' is here used to cover topology design where several physical phenomena are involved in the problem statement, thus covering situations where for example elastic, thermal and electromagnetic analyses are involved. When modelling such situations, the basic concept of the density distribution method for topology design provides a general framework for computations, but here the initial obstacle is the need for interpolation of not only stiffness but also other physical properties. If only linear models are considered, one possibility is to use the theory and computational framework of homogenization of composite media to compute effective elastic, thermal and electromagnetic properties of a given type of composites and use such relationships between intermediate density and material properties in the design problem. An example of this approach for thermo-elastic problems can be found in [30]. However, the less complex design description of the SIMP approach has lead also 

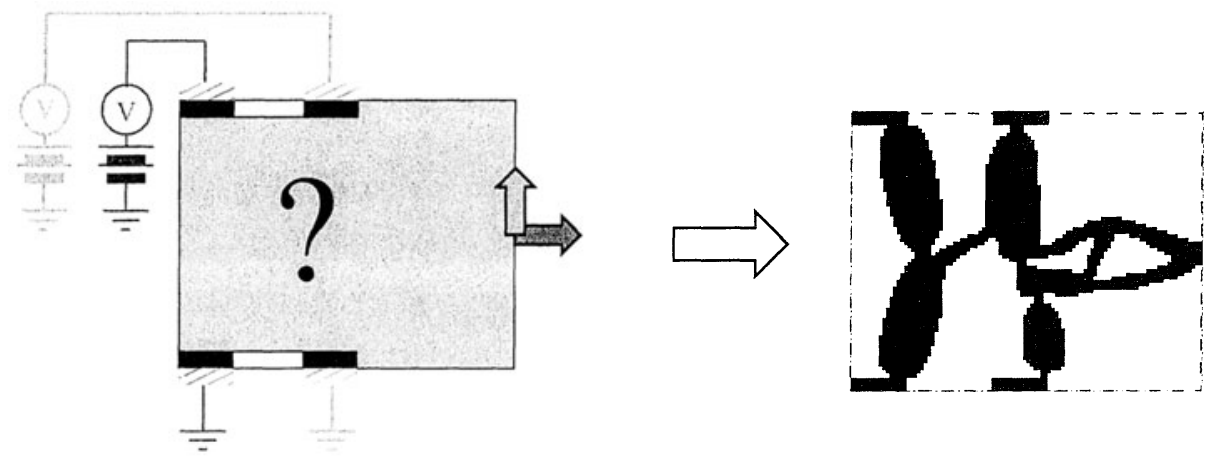

Figure 6 Design of a thermo-electro-mechanical actuator. The mid right-hand point should move right or up when voltage is applied at the corresponding poles. The movement is through elastic deformation due to the temperature change which occurs when a current passes through the structure. By courtesy of O. Sigmund, [35]

to the development of such interpolation schemes for multiple physics problems. As an example of this, in reference [37], microstructures with extreme thermal expansion are designed by combining a material interpolation of elastic properties with a similar interpolation of the thermal expansion coefficients.

\section{DIFFICULTIES - FUTURE WORK}

The models described here all refer to an approach to topology design where material is distributed in a fixed domain. A pivotal aspect of this idea in computational implementations is the use of a fixed FEM mesh for the domain. This is not an inherent requirement, but is useful for computational efficiency. If a topology of material and void is the goal of the design process, this will imply that low density areas are also included in the analysis for each feasible design. For stress constraints this leads to the difficulty of the so-called 'stress singularity phenomenon', where low density regions may have high stress but are structurally insignificant for the final design, and where regularization (in the sense of mathematical programming) is needed for numerically solving such problems ([14]). A similarly elusive problem arising from this basic design representation appears in situations involving stability and vibration criteria. The relevant data to consider in such situations are the eigenvalues of the structurally relevant parts of the structure, i.e., the buckling loads and the vibration frequencies of the 'black' part of a black- and-white design. In a true black-and-white design this are the non-zero eigenvalues, but at intermediate steps of an iterative opti- 
mization method implemented with interpolation schemes it can become unclear what are the relevant values to consider. Examples of this are localized modes which appear in low density regions and which should be filtered out in order that the optimization deals with the structurally interesting modes. Moreover, one should cater for certain aspects which complicates modelling, for example that no structure what-so-ever has the highest eigenfrequency of all structures with only structural mass. See references $[24,26]$ for further discussion.

For future work it would be beneficial to reconsider the way geometry representation is currently handled. This should include studying alternatives to the current close linking of geometry and physics where the same finite element grid is used to approximate the geometry and the mechanical response fields. Here grid-less methods may prove useful. Typically, in fixed domain material distribution methods, the grid is a uniform, rectangular partition of space and the design variables are assumed to be constant within each element. Thus, a raster model is imposed on the geometry. This approach has a number of very beneficial features in terms of computational efficiency, but it also has some intrinsic less than desirable features (non-smooth boundary representation etc.) which are typically not too severe if they are recognized and addressed in the implementation as well as in the interpretation of the computer generated designs. However, as outlined above for the stress constrained and vibration constrained problems, the fixed grid representation for geometry and analysis implies that it can be rather tricky to handle the correct formulation of objectives and constraints as well as interpolating physical data for intermediate densities, a feature which gets exaggerated when considering non- linear material behaviour. The latter could be circumvented by further success in handling the 0-1 problem directly, an area of utmost importance. For the problems arising due to the fictitious domain type approach to analysing on a fixed domain a new approach in the area of topology design may be the only option. In view of this, it seems that future research could also benefit from investigations into alternative approaches to topology design for blackand-white design, with an emphasis on the geometric modelling aspect. Here the concept of the bubble method ([15]) should no doubt receive further attention, especially in the light of recent work on the concept of a general topological derivative, see [38]. Work in this direction could also be helpful in an effort to rejuvenate the field of optimal boundary shape design, an important area, unfortunately currently lingering an idle life. 


\section{USING THE WEB}

Much useful information on topology design can be found on the Internet. A free topology optimization test-site (make your own design!) can be found at http://www.topopt.dtu.dk/. Here one can also find tutorials on the subject, a free 99 line MATLAB topology optimization code, as well as interesting links to academic and commercial sites.

\section{Acknowledgments}

The author would like to thank Ole Sigmund for permission to use several illustrations shown in this presentation. Kind assistance by Jens Gravesen when preparing this paper is also gratefully acknowledged.

\section{References}

[1] G. Allaire, E. Bonnetier, G. Francfort and F. Jouve (1997), Shape optimization by the homogenization method, Numerische Mathematik, 76, pp. 27-68.

[2] G. Allaire and R. Kohn (1993a), Optimal bounds on the effective behaviour of a mixture of two well-ordered elastic materials, Quaterly of Appled Mathematics, 51, pp. 643-674.

[3] G. Allaire and R. Kohn (1993b), Optimal design for minimum weight and compliance in plane stress using extremal microstructures, European Journal of Mechanics A, 12, pp. 839-878.

[4] L. Ambrosio and G. Buttazzo (1993), An optimal design problem with perimeter penalization, Calculus and Variation, 1, pp. 55-69.

[5] A. Back-Pedersen (1999), Taking advantage of using both topology and shape optimization for practical design, in Proc. NAFEMS World Congress '99, Newport, Rhode Island, NAFEMS ltd, pp. 889899.

[6] M. Beckers (1999), Topology optimization using a dual method with discrete variables, European Journal of Mechanics A, 17, pp. $14-24$.

[7] M. Bendsøe (1995), Optimization of Structural Topology, Shape, and Material, Springer Verlag, Heidelberg.

[8] M. Bendsøe and O. Sigmund (1999), Material interpolation schemes in topology optimization, Archives of Applied Mechanics, 69, pp. 635-654.

[9] M. Bendsøe and N. Kikuchi (1988), Generating optimal topologies in structural design using a homogenization method, Computer Methods in Applied Mechanics and Engineering, 71, pp. 197224. 
[10] B. Bourdin (1999), Filters in topology optimization, Danish Center for Applied Mathematics and Mechanics, DCAMM Report No. 628.

[11] G. Cheng and N. Olhoff (1981)], An investigation concerning optimal design of solid elastic plates, International Journal of Solids and Structures, 17, pp. 305-323.

[12] A. Díaz and N. Kikuchi (1992), Solutions to shape and topology eigenvalue optimization problems using a homogenization method, International Journal for Numerical Methods in Engineering, 35, pp. 1487-1502.

[13] A. Diaz and R. Lipton (1997), Optimal material layout for 3D elastic structures, Structural Optimization, 13, pp. 60-64.

[14] P. Duysinx and M. Bendsøe (1998), Topology optimization of continuum structures with local stress constraints, International Journal for Numerical Methods in Engineering, 43, pp. 1453-1478.

[15] H. Eschenauer, V. Kobelev and A. Schumacher (1994), Bubble method of topology and shape optimization of structures, Structural Optimization, 8, pp. 42-51.

[16] C. Fleury (1989), CONLIN: an efficient dual optimizer based on convex approximation concepts, Structural Optimization, 1, pp. 8189 .

[17] J. Goodman, R. Kohn and L. Reyna (1986), Numerical study of a relaxed variational problem from optimal design, Computer Methods in Applied Mechanics and Engineering, 57, pp. 107-127.

[18] M. Kamat (1995), Structural Optimization - Status and Promise, AIAA, Washington D.C.

[19] N. Kikuchi, S. Nishiwaki, J. Fonseca and E. Silva (1998), Design optimization method for compliant mechanisms and material microstructure, Computer Methods in Applied Mechanics and Engineering, 151, pp. 401-417.

[20] R. Kohn and G. Strang (1986), Optimal design and relaxation of variational problems, Communications in Pure and Applied Mathematics, 39, pp. 113-137, pp. 139-182, pp. 353-377.

[21] U. Larsen, O. Sigmund and S. Bouwstra (1997), Design and fabrication of compliant mechanisms and material structures with negative poissons ratio, Journal of MicroElectroMechanical Systems, 6, pp. 99-106.

[22] J.-L. Lions (1981), Some Methods in the Mathematical Analysis of Systems and Their Control, Gordon and Breach Science Publishers, Inc., New York. 
[23] K. Lurie, A. Cherkaev and A. Fedorov (1982), Regularization of optimal design problems for bars and plates, I, II, III, Journal of Optimization Theory and Applications, 37, pp. 499-543, 42, pp. 247-282.

[24] M. Neves, H. Rodrigues and J. Guedes (1995), Generalized topology design of structures with a buckling load criterion, Structural Optimization, 10, pp. 71-78.

[25] J. Ou and N. Kikuchi (1996), Optimal design of controlled structures, Structural Optimization, 11, pp. 19-28.

[26] N.L. Pedersen (1999), Maximization of eigenvalues using topology optimization, Danish Center for Applied Mathematics and Mechanics, DCAMM Report No. 620.

[27] J. Petersson (1999a), A finite element analysis of optimal variable thickness sheets, SIAM Journal of Numerical Analysis, 36, pp. 1759-1778.

[28] J. Petersson (1999b), Some convergence results in perimetercontrolled topology optimization, Computer Methods in Applied Mechanics and Engineering, 171, pp. 123-140.

[29] J. Petersson and O. Sigmund (1998), Slope constrained topology optimization, International Journal for Numerical Methods in Engineering, 41, pp. 1417-1434.

[30] H. Rodrigues and P. Fernandes (1995), A material based model for topology optimization of thermoelastic structures, International Journal for Numerical Methods in Engineering, 38, pp. 1951-1965.

[31] G. Rozvany (1997), Topology Optimization in Structural Mechanics, Springer Verlag, Heidelberg.

[32] U. Schramm (1999), The use of structural optimization in automotive design - state of the art and vision, in Bloembaum, C., editor, Proc. Third World Congress of Structural and Multidisciplinary Optimization, University of New York at Buffalo, pp. 200-202.

[33] O. Sigmund (1995), Tailoring materials with prescribed elastic properties, Mechanics of Materials, 20, pp. 351-368.

[34] O. Sigmund (1997), On the design of compliant mechanisms using topology optimization, Mechanics of Structures and Machines, 25, pp. $495-526$.

[35] O. Sigmund (1998), Topology optimization in multiphysics problems, in Proc. 7th AIAA/USAF/NASA/ISSMO Symposium on Multidisciplinary Analysis and Optimization St. Louis MI Sept. 2-4 '98, AIAA, pp. 1492-1500. 
[36] O. Sigmund and J. Petersson (1998), Numerical instabilities in topology optimization, Structural Optimization, 16, pp. 68-75.

[37] O. Sigmund and S. Torquato (1997), Design of materials with extreme thermal expansion using a three-phase topology optimization method, Journal of the Mechanics and Physics of Solids, 45, pp. 1037-1067.

[38] J. Sokolowski and A. Zochowski (1999), On the topological derivative in shape optimization, SIAM Journal on Control and Optimization, 37, pp. 1251-1272.

[39] K. Svanberg (1987), The method of moving asymptotes - a new method for structural optimization, International Journal for $\mathrm{Nu}$ merical Methods in Engineering, 24, pp. 359-373.

[40] R. Yang and A. Chahande (1995), Automotive applications of topology optimization, Structural Optimization, 9, pp. 245-249.

[41] J. Zowe, M. Kocvara and M. Bendsøe (1997), Free material optimization via mathematical programming, Mathematical Program$\operatorname{ming} B, 79$, pp. 445-466. 